\title{
Editorial Comment to "Evaluating the Oncological Outcomes of Pure Laparoscopic Radical Nephroureterectomy Performed for Upper Tract Urothelial Carcinoma Patients: A Multicenter Cohort Study Adjusted by Propensity Score Matching”
}

\author{
Tsunenori Kondo, MD, PhD \\ Department of Urology, Tokyo Women's Medical University Medical Center East, Tokyo, Japan
}

Bladder cuff resection is currently considered a crucial step in radical nephroureterectomy (RNU), which influences the oncological outcome of patients with upper tract urothelial carcinoma (UTUC). ${ }^{1}$ Laparoscopic RNU (LRNU) has been widely performed to reduce the surgical invasiveness of patients with UTUC. It would be ideal if the entire RNU procedure could be performed laparoscopically; however, laparoscopic management of the bladder cuff is technically difficult, and thus the bladder cuff is often resected using the open procedure after laparoscopic mobilization of the kidney (conventional LRNU). In addition, there is a risk of deteriorating oncological outcome in laparoscopic management of the bladder cuff because of incomplete resection. ${ }^{2}$ Hence, the oncological outcome of pure LRNU, in which the management of the bladder cuff is also performed laparoscopically, needs to be clarified with a large number of patients.

Shigeta et al. conducted a retrospective study comparing pure LRNU and conventional LRNU in their propensityscore matched cohort. ${ }^{3}$ Their results revealed a higher risk of intravesical tumor dissemination from pure LRNU than from conventional LRNU. In addition, the pure LRNU group showed atypical recurrence in some patients due to peritoneal dissemination, and a lower recurrence-free rate

(C) Society of Surgical Oncology 2020

First Received: 7 August 2020

Accepted: 13 August 2020;

Published Online: 18 August 2020

T. Kondo, MD, PhD

e-mail: tkondo@twmu.ac.jp compared with the conventional LRNU groups, although statistical difference was not found. The study by Shigeta et al. was not a prospective randomized study, but nonetheless it appears to provide useful information for physicians selecting the surgical approach in patients with UTUC. However, some points need to be discussed.

One of these major points is a technical issue. According to the authors, they performed the bladder cuff resection by sharp dissection around the intramural ureter, followed by two-layer closure of the bladder in pure LRNU. This raises the question as to why pure laparoscopic management is more likely to cause tumor dissemination inside and outside of the bladder when compared with open resection. The authors speculated some possible explanations, including (1) excessive manipulation of the bladder; (2) prolonged pneumoperitoneum pressure; and (3) a transperitoneal approach. This is similar to a robot-assisted radical cystectomy (RARC), where the risk of peritoneal dissemination has been noted in early reports. ${ }^{4}$ However, subsequent studies have failed to find a higher incidence of peritoneal dissemination from RARC; ${ }^{5}$ thus, the tumor spillage may be associated with an unrefined technique during the learning curve of new surgical procedures. This may account for the pure LRNU results in the present study.

Another important point is patient selection. The authors described that the selection procedure for performing either pure LRNU or conventional LRNU was completed according to the surgeons' preference and the patients' request. Electronic supplementary Fig. 1 shows that half of the patients have undergone pure LRNU in the last few years. Although it is unclear how many surgeons can perform pure LRNU in their institutes, I believe that only a 
few experienced surgeons are able to perform this procedure. These surgeons have been applying this technique for more challenging cases, such as those with obesity or a small pelvic space. This might result in incomplete resection of the bladder cuff or closure of the ureteral stump, which could cause inferior oncological outcomes of pure LRNU.

The most important point is how they will change their practice patterns according to the results of this study. We have not performed pure LRNU in our institutes since there was a technical hurdle on the pure laparoscopic management of the bladder cuff, as previous studies have already demonstrated. ${ }^{2}$ Moreover, it is not easy to perform lymphadenectomy laparoscopically. In fact, a small incision has to be made to extract the resected specimens, even in pure LRNU. From a clinical standpoint, I believe that there is only a minimal difference between approaches regarding their invasiveness. Whether the practice of this technique will continue remains unclear. If so, surgeons obviously need to refine their technique to prevent tumor spillage. Moreover, adjuvant chemotherapy was performed in only approximately $20 \%$ of patients in this study. A recent study shows adjuvant chemotherapy may improve the chances of survival; ${ }^{6}$ thus, it would be interesting to examine whether it also plays a role in preventing atypical recurrence.

Nevertheless, the present study has proposed a very important message-that the oncological outcomes of patients with UTUC is definitely influenced by not only whether or not the bladder cuff is resected but also how it is resected. We have been entering a new era of robotic surgery, where surgical precision should be considered increasingly important. In fact, robotic RNU (RRNU) has been adopted as a new, less invasive surgery for patients with UTCU in Western countries. I am sure that RRNU will be dramatically substituted for LRNU in the near future. During RRNU, a similar technique has been used for the management of bladder cuff resection. We believe that robotic management of the bladder cuff resection would be more precise than the laparoscopic approach. To the best of my knowledge, there have been no reports of an increased risk of atypical recurrence associated with RRNU. ${ }^{7}$ However, as was shown in the present study, we should note that atypical recurrence may occur, especially during the learning curve of RRNU, and steps should be taken to not compromise the oncological outcomes of patients.

DISCLOSURE Tsunenori Kondo received honoraria from Pfizer and Ono Pharmaceutical.

\section{REFERENCES}

1. Roupret M, Babjuk M, Comperat E, et al. European Association of Urology Guidelines on Upper Urinary Tract Urothelial Carcinoma: 2017 Update. Eur Urol. 2018;73(1):111-122.

2. Peyronnet B, Seisen T, Dominguez-Escrig JL, et al. Oncological Outcomes of Laparoscopic Nephroureterectomy Versus Open Radical Nephroureterectomy for Upper Tract Urothelial Carcinoma: An European Association of Urology Guidelines Systematic Review. Eur Urol Focus. 2019;5(2):205-223.

3. Shigeta K, Matsumoto K, Takeda $\mathrm{T}$, et al. Evaluating the Oncological Outcomes of Pure Laparoscopic Radical Nephroureterectomy performed for Upper-tract Urothelial Carcinoma Patients: A Multicenter Cohort Study Adjusted by Propensity Score Matching. Ann Surg Oncol. 2020. https://doi. org/10.1245/s10434-020-09046-9.

4. Nguyen DP, Al Hussein Al Awamlh B, Wu X, et al. Recurrence patterns after open and robot-assisted radical cystectomy for bladder cancer. Eur Urol. 2015;68(3):399-405.

5. Faraj KS, Abdul-Muhsin HM, Rose KM, et al. Robot Assisted Radical Cystectomy vs Open Radical Cystectomy: Over 10 years of the Mayo Clinic Experience. Urol Oncol. 2019;37(12):862-869.

6. Birtle A, Johnson M, Chester J, et al. Adjuvant chemotherapy in upper tract urothelial carcinoma (the POUT trial): a phase 3, openlabel, randomised controlled trial. The Lancet. 2020;395(10232):1268-1277.

7. Veccia A, Antonelli A, Francavilla S, et al. Robotic versus other nephroureterectomy techniques: a systematic review and metaanalysis of over 87,000 cases. World J Urol. 2020;38(4):845-852.

Publisher's Note Springer Nature remains neutral with regard to jurisdictional claims in published maps and institutional affiliations. 